Ischaemic and haemorrhagic stroke

\section{Shared mechanisms of ischaemic and haemorrhagic stroke: still a lot to learn}

\section{P Michel, J Bogousslavsky}

\section{Ischaemic and haemorrhagic strokes may have a common pathogenesis}

$\mathrm{T}$ risk of intracerebral haemorrhage (ICH) is increased in patients with ischaemic cerebrovascular disease and vice versa. ${ }^{1}$ Risk factors shared by both stroke subtypes, such as hypertension, age, and smoking, partly explain this observation. While some researchers stress that most ischaemic stroke subtypes have similar risk factor profiles, one can go further and claim that most strokes (ischaemic and haemorrhagic) are the result of a common final pathway: arterial wall damage with accumulation of abnormal cells and proteins, inflammation, molecular changes, and eventual breakdown of the intima and media. Hypertensive cerebral arteriolopathy and cerebral amyloid angiopathy are examples where ischaemic and haemorrhagic brain damage frequently coexist. Leukoaraiosis and microbleeds may both be markers of a single disease process affecting small cerebral arteries, putting patients at risk of both ischaemia and haemorrhages. This double risk may also explain why aggressive antithrombotic treatments fail in ischaemic stroke patients: the haemorrhage rate matches ${ }^{2}$ or exceed $s^{3}$ the reduction in ischaemic events.

Does the study by Ariesen et al in this issue of the journal (see pages 92-4) support the hypothesis that ischaemic and haemorrhagic strokes have a common pathogenesis? The authors found that in patients with previous ischaemic strokes, older age, hypertension, and current antihypertensive treatment are associated with a subsequently increased ICH risk. Most of their patients were on antithrombotic treatment, although the exact numbers are not stated. These results strongly support the presence of shared mechanisms of ischaemic and haemorrhagic events.

The finding that higher blood glucose levels were associated with decreased
ICH rates is unexpected. It is at odds with a previous meta-analysis by the same authors, but not with our recent observations. ${ }^{4}$ Why should diabetics who have had an ischaemic stroke be more resistant to ICH? They have more white matter ischaemia, brain atrophy, and cognitive decline than non-diabetics. ${ }^{45}$ Some of this is likely due to pathology in small arteries, but it remains unknown whether the arteriolopathy of diabetics without hypertension is different from the arteriolopathy in hypertensive patients. Even if the protective effects of hyperglycaemia are confirmed by other studies, this by no means allows physicians to liberally prescribe antithrombotics: in a randomised controlled trial where $68 \%$ of patients were diabetic, combination antiplatelet treatment increased ICH significantly. ${ }^{2}$

Which patients are at very high risk of haemorrhage after ischaemic stroke and may therefore be poor candidates for antithrombotic treatment? Based on the findings by Ariesen et al, one might conclude that elderly hypertensive patients should not receive antithrombotic treatment after an ischaemic stroke. Unfortunately, hypertension and age are not only major risk factors for ICH but also for recurrence of ischaemic stroke. As recurrences after ischaemic stroke are mostly ischaemic, ${ }^{1}$ withholding antithrombotics in such patients will likely allow more ischaemic events to occur than it will avoid haemorrhages. Adding other markers that were not studied by Ariesen et al, such as alcohol intake, cognitive decline, leukoaraiosis, or microbleeds, may allow more precise definition of a very high risk group in the future. But again, only randomised controlled trials on the risk of antithrombotics in such patients will be able to answer these questions.
Fortunately, several interventions have been shown to simultaneously reduce ischaemic and haemorrhagic strokes, probably by reducing damage to arterial walls: blood pressure lowering in hypertensive, normotensive, and elderly patients, ${ }^{67}$ cessation of smoking, ${ }^{8}$ and regular physical activity. ${ }^{9}$ Until more is known, physicians will have to strictly apply this knowledge to prevent stroke recurrences. For patients suffering from both ischaemic and haemorrhagic cardiocerebrovascular events, use of antithrombotics will still have to be decided upon on an individual basis, given the lack of evidence that may last for many more years to come.

J Neurol Neurosurg Psychiatry 2006;77:1. doi: 10.1136/jnnp.2005.078287

\section{Authors' affiliations}

P Michel, J Bogousslavsky, Centre Hospitalier Universitaire Vaudois, Lausanne, Switzerland

Correspondence to: Dr Patrik Michel, Centre Hospitalier Universitaire Vaudois, Rue du Bugnon 46, 1011 Lausanne, Switzerland Patrik.Michel@chuv.ch

Competing interests: none declared

\section{REFERENCES}

1 Yamamoto H, Bogousslavsky J. Mechanisms of second and further strokes. J Neurol Neurosurg Psychiatry 1998;64(6):771-6.

2 Diener HC, Bogousslavsky J, Brass LM, et al. Aspirin and clopidogrel compared with clopidogrel alone after recent ischaemic stroke or transient ischaemic attack in high-risk patients (MATCH): randomised, double-blind, placebo-controlled trial. Lancet 2004;364(9431):331-7.

3 Chimowitz MI, Lynn MJ, Howlett-Smith $\mathrm{H}$, et al. Comparison of warfarin and aspirin for symptomatic intracranial arterial stenosis. N Engl J Med 2005;352(13):1305-16.

4 Karapanayiotides T, Piechowski-Jozwiak B, van Melle G, et al. Stroke patterns, etiology, and prognosis in patients with diabetes mellitus. Neurology 2004;62(9):1558-62.

5 Schmidt R, Launer L, Nilsson LG, et al. Magnetic resonance imaging of the brain in diabetes: the Cardiovascular Determinants of Dementia (CASCADE) Study. Diabetes 2004;53(3):687-92

6 PROGRESS Collaborative Group. Randomised trial of a perindopril-based blood-pressurelowering regimen among 6,105 individuals with previous stroke or transient ischaemic attack. Lancet 2001;358(9287): 1033-41.

7 Staessen JA, Gasowski J, Wang JG, et al. Risks of untreated and treated isolated systolic hypertension in the elderly: meta-analysis of outcome trials. Lancet 2000;355(9207):865-72

8 Straus SE, Majumdar SR, McAlister FA. New evidence for stroke prevention: scientific review. JAMA 2002;288(1 1):1388-95.

9 Lee CD, Folsom AR, Blair SN. Physical activity and stroke risk: a meta-analysis. Stroke 2003;34(10):2475-81. 


\section{Multiple sclerosis related fatigue G Giovannoni}

\section{Fatigue is often the most disabling symptom of MS}

$\mathrm{H}$ ow we define fatigue remains as controversial today as it did 40 years ago: "True fatigue and....tiredness are plainly different." ${ }^{1}$. Fatigue is more than tiredness and has recently been referred to as "pathological exhaustion". 2 In this context the term "pathological" would, for example, classify the physical fatigue which athletes experience as part of voluntary effort as being abnormal. Fatigue must therefore surely be a normal phenomenon-a subjective feeling of tiredness or exhaustion which could refer to both physical (motor activities) and mental (cognitive or emotional) processes. Fatigue is only pathological if it is disabling - that is, if it affects a person's social, physical, and occupational wellbeing. For lack of a better definition the Centres for Disease Control and Prevention (CDC) defines "profound fatigue" and by implication "pathological fatigue" in the context of the chronic fatigue syndrome (CFS) as fatigue that "is not improved by bed rest and that may be worsened by physical or mental activity". ${ }^{3}$ This definition appropriately excludes exercise induced or temperature dependent conduction block, a form of physical fatigue which occurs in subjects with demyelinating disease.

Approximately $80 \%$ of subjects with multiple sclerosis (MS) have pathological fatigue and in half these cases it is their most disabling symptom. In general, fatigue does not correlate with neurological impairment, physical disability, or the lesion load on conventional magnetic resonance imaging (MRI). In one study, subjects with "benign MS" had as much fatigue as those with non-benign MS. ${ }^{4}$ Rarely, MS may present initially as chronic fatigue. In more recent studies a relation has been demonstrated between altered cerebral activation patterns ${ }^{5}$ and the development of progressive brain atrophy ${ }^{6}$ and fatigue. In the latter study the development of progressive brain atrophy was independent of disability, mood, or other MRI findings. ${ }^{6}$ These observations question whether anatomical and physiological substrates underlie MS related fatigue.

One emerging hypothesis is that MS related fatigue is caused by ongoing inflammation. Fatigue that occurs as a result of acute infections can be reproduced by the administration of proinflammatory cytokines-for example, the type 1 interferons ( $\alpha$ or $\beta$ ) or interleukin 2. The evolutionary benefits of fatigue in relation to systemic infection are obvious - in response to an infection the immune response triggers a behavioural response to maximise an animal's chance of recovery and hence of survival. Thus it is not surprising that fatigue is such a prominent symptom in chronic diseases associated with systemic inflammation. In rheumatoid arthritis, a systemic inflammatory disease without obvious CNS pathology, levels of acute phase proteins correlate with fatigue. ${ }^{7}$ In MS, weak correlations between fatigue and markers of systemic inflammation have been reported. ${ }^{4}$ Similarly, in this issue of the journal (see pages 34-9), Heesen et al report a weak association between fatigue and the stimulated whole blood production of the proinflammatory cytokines tumour necrosis factor $\alpha$ (TNF $\alpha)$ and interferon $\gamma$ (IFN $\gamma)$. ${ }^{9}$ An important observation, however, is that MS related fatigue does not correlate with Gd enhancing lesions on MRI, ${ }^{6}{ }^{10}$ the most widely accepted marker of active inflammation in MS. These observations suggest that MS related fatigue is linked to peripheral rather than central inflammation. This may explain why treatment with IFN $\beta$, a systemically administered cytokine that reduces MRI activity, is not associated with an improvement in fatigue scores. ${ }^{11}$ How systemic inflammation induces fatigue is unknown, but the symptom may be mediated by proinflammatory cytokines, similar to the hypnotic effects of interleukin 1 and TNF $\alpha .^{12}$

Whether or not fatigue is a sensory percept is a moot point. Focal areas of the cerebral cortex or subcortical structures involved in the perception of fatigue have not been identified. On the other hand, fatigue and arousal may have a similar neuroanatomical basis. In arousal, subcortical systems integrate sensory and environmental information, which is processed and stimulates the cerebral cortex through the ascending reticular activating system. The latter

An important caveat to the above observations and to the ongoing research into MS related fatigue is the lack of well validated outcome measures to quantify fatigue. The interpretation of fatigue by subjects responding to the most commonly used fatigue questionnaires may be context and disease specific. Work is therefore required to standardise the measurement of fatigue in MS and other conditions.

Uncertainties over the definition, pathogenesis, and measurement of MS related fatigue are clearly hampering the testing of specific therapeutic antifatigue strategies. There are, however, unproven strategies that could be tried to help ameliorate MS related fatigue. As fatigue appears to be related to mood and quality of life, it is important to address these issues at the outset. Similarly, it is important to exclude common medical conditions that could exacerbate fatigue-for example hypothyroidism-and to optimise the doses of drugs that are known to exacerbate fatigue. Non-pharmacological approaches for the specific management of fatigue include behavioural therapy, graded aerobic exercise programmes, energy conservation strategies, dietary advice, environmental cooling, and improvement in basic sleep hygiene. For subjects experiencing disabling fatigue, amantadine may be partially effective. ${ }^{13}$ Despite its widespread use, modafinil has yet to be shown to be effective in MS related fatigue. ${ }^{14}{ }^{15}$ Antidepressant drugs, particularly serotonin and the noradrenaline reuptake inhibitors, are widely used, with little or no evidence to support their effectiveness in MS related fatigue. The use of stimulants-such as amphetamines, methylphenidate, and pemoline (discontinued in the UK) - in MS related fatigue cannot be sanctioned in view of their unproven efficacy and potential side effects.

If the findings of Heesen et al prove to be correct then targeting inflammation may be the most effective anti-fatigue strategy in MS. Glatiramer acetate, which has an impact on MS relapses similar to IFN $\beta$ but which is not associated with the flu-like symptoms, appears to have a positive impact on MS related fatigue. ${ }^{11}$ Nataluzimab, a selective adhesion molecule antagonist which reduces the relapse rate in MS by more than $50 \%$, markedly improved the perception of wellbeing (a crude index that includes fatigue) compared with placebo. ${ }^{16}$

It is important that we, as health care workers, should recognise and understand the impact that MS related fatigue 
has on sufferers from MS, and be aware of the emerging evidence that at least a component of this complex symptom is linked to inflammatory disease activity and that strategies are emerging to manage fatigue more effectively.

J Neurol Neurosurg Psychiatry 2006;77:2-3. doi: 10.1136/jnnp.2005.074948

Correspondence to: Dr Gavin Giovannoni, Department of Neuroinflammation, Institute of Neurology, University College London, Queen Square, London WCIN 3BG, UK; g.giovannoni@ion.ucl.ac.uk

Competing interests: none declared

\section{REFERENCES}

1 Editorial. Fatigue. Lancet, 1966;i, 585-6.

2 Barnett R. Fatigue. Lancet 2005;366:21.
3 Website. Chronic fatigue syndrome (http://www. cdc.gov/ncidod/diseases/cfs/about/what.htm).

4 Giovannoni G, Thompson AJ, Miller DH, et al. Fatigue is not associated with raised inflammatory markers in multiple sclerosis. Neurology 2001;57:676-81.

5 Filippi M, Rocca MA, Colombo B, et al. Functional magnetic resonance imaging correlates of fatigue in multiple sclerosis. Neuroimage 2002; 15:559-67.

6 Marrie RA, Fisher E, Miller DM, et al. Association of fatigue and brain atrophy in multiple sclerosis. I Neurol Sci 2005;228:161-6.

7 Elliott MJ, Maini RN, Feldmann M, et al. Randomised double-blind comparison of chimeric monoclonal antibody to tumour necrosis factor alpha (cA2) versus placebo in rheumatoid arthritis. Lancet 1994;344:1105-10.

8 Flachenecker $\mathrm{P}$, Bihler I, Weber F, et al. Cytokine mRNA expression in patients with multiple sclerosis and fatigue. Mult Scler 2004;10:165-9.

9 Heeson C, Nawrath L, Reich C, et al. Fatigue in multiple sclerosis. J Neurol Neurosurg Psychiatry 2005:75:34-9.

10 Mainero C, Faroni J, Gasperini C, et al. Fatigue and magnetic resonance imaging activity in multiple sclerosis. J Neurol 1999;246:454-8.

11 Metz LM, Patten SB, Archibald CJ, et al. The effect of immunomodulatory treatment on multiple sclerosis fatigue. I Neurol Neurosurg Psychiatry 2004;75:1045-7.

12 Krueger JM, Majde JA. Humoral links between sleep and the immune system: research issues. Ann NY Acad Sci 2003:992:9-20.

13 Taus C, Giuliani G, Pucci E, et al. Amantadine for fatigue in multiple sclerosis. Cochrane Database Syst Rev 2003:CD002818.

14 Rammohan KW, Rosenberg JH, Lynn DJ, et al. Efficacy and safety of modafinil (Provigil) for the treatment of fatigue in multiple sclerosis: a two centre phase 2 study. J Neurol Neurosurg Psychiatry 2002;72:179-83.

15 Stankoff B, Waubant E, Confavreux C, for the French Modafinil Study Group, et al. Modafinil for fatigue in MS: a randomized placebocontrolled double-blind study. Neurology 2005;64: 1139-43.

16 Miller DH, Khan OA, Sheremata WA, for the International Natalizumab Multiple Sclerosis Trial Group, et al. A controlled trial of natalizumab for relapsing multiple sclerosis. N Engl J Med 2003:348:15-23.

\section{"One person yawning sets off everyone else"}

\section{M-Pierre Perriol, C Monaca}

\section{The precise role of yawning in human physiology remains unclear}

Y awning is a stereotyped behaviour present in most mammals from rodents to humans and has been described since antiquity. Hippocrates considered yawning to be an exhaustion of the fumes preceding fever. Modern medicine did not pay much attention to it until the 1980s, when, with advances in neuropharmacology, yawning proved to be a valuable tool for the assessing dopaminergic activity and the pharmacological properties of new drugs. However, its precise role in human physiology is still unknown and its mechanisms remain unclear. The paper by Cattaneo et al (see pages 98-100) reports two cases of pathological yawning as the earliest symptom of brain stem infarction which introduces new arguments for locating this neuronal network in the lower brain stem.

Yawning occurs after waking up, before eating, before sleeping, and in passive activities when it is necessary to maintain a certain level of vigilance. ${ }^{1}$ It is then followed by an acceleration of the electroencephalographic rhythms. It does not serve a primary respiratory function and it clearly has a non-verbal communicative status. Nevertheless, it is also a clinical sign in intracranial hypertension, migraine, or iatrogenic side effects of dopaminergic drugs and serotonin reuptake inhibitors. ${ }^{2}$ In basal ganglia disorders, yawning is reduced in patients with Parkinson's disease, and occurs more often in patients with Huntington's disease and supranuclear palsy than in controls. In healthy volunteers, apomorphine induces yawning which is also observed at the beginning of the "on" periods in Parkinson's disease. ${ }^{2}$

The anatomical structures known to be implicated in the occurrence and control of yawning are the paraventricular nucleus of the hypothalamus $(\mathrm{PVN})$, the hippocampus, the reticular formation, the neostriatum, and the cranial (V, VII, IX, X, XI, XII), cervical $(\mathrm{Cl}-\mathrm{C} 4)$, and dorsal nerves. Yawning is probably a reflex answer of the brain stem reticular formation aimed to increase the cortical level of vigilance. Dopamine and oxytocin are the main neurotransmitters implicated in its modulation. Indeed yawning induces sensory efferents from the terminals of the fifth facial nerve to the reticular formation or the PVN through the spinothalamic and hypothalamic tracts. Stimulation of the dopamine D2 receptors of the PVN activates the oxytocin neurones that project either to the pons (reticular formation, locus coeruleus), to the hippocampus, to the insula, or to the orbitofrontal cortex, leading to the transient feeling of wellbeing that follows yawning. This pathway is modulated by acetylcholine, serotonin, opioid peptides, sexual hormones, and orexin. The paper by Cattaneo et al provides important data on the crucial role of the lower brain stem.

Contagious yawning is an even more intriguing phenomenon. It is triggered by seeing, hearing, or even thinking about someone else yawning. Contagious yawning does not occur in species that do not recognise themselves in mirrors or in infants younger than two years old. The phenomenon has been investigated with functional magnetic resonance imaging, ${ }^{3}$ which implicated the precuneus or the posterior cingulate regions, functional regions associated with the identification of self referent information, a primitive form of empathy. Further studies are needed before conclusions can be drawn.

J Neurol Neurosurg Psychiatry 2006;77:3. doi: 10.1136/jnnp.2005.078337

\section{Authors' affiliations}

M-P Perriol, C Monaca, Department of Clinical Neurophysiology, Lille University Hospital, Lille, France

Correspondence to: Dr Marie-Pierre Perriol, mperriol@yahoo.fr

Competing interests: none declared

\section{REFERENCES}

1 Daquin G, Micallef J, Blin O. Yawning. Sleep Med Rev 2001;5, 299-312.

2 Walusinski O, Depute BL. Le bâillement: phylogénèse, étiologie, nosologie. Rev Neurol 2004;160:1011-121.

3 Platek SM, Mohamed FN, Gallup GG. Contagious yawning and the brain. Cogn Brain Res 2005;23:448-52. 